\title{
Terapia On-line com Casais e Famílias: Prática e Formação na Pandemia de Covid-19
}

\author{
Beatriz Schmidt ${ }^{1}$ \\ ${ }^{1}$ Universidade Federal do Rio Grande, RS, Brasil. \\ Isabela Machado da Silva ${ }^{2}$ \\ ${ }^{2}$ Universidade de Brasília, DF, Brasil.
}

\author{
Maria Adélia Minghelli Pieta ${ }^{3}$ \\ ${ }^{3}$ Universidade Federal do Rio Grande do Sul, RS, Brasil. \\ Maria Aparecida Crepaldi ${ }^{4}$ \\ ${ }^{4}$ Universidade Federal de Santa Catarina, SC, Brasil.
}

\author{
Adriana Wagner ${ }^{3}$ \\ ${ }^{3}$ Universidade Federal do Rio Grande do Sul, RS, Brasil.
}

\begin{abstract}
Resumo: A pandemia de Covid-19 potencializou o já emergente processo de intensificação das conexões virtuais. Para terapeutas de casal e família, essa grave crise de saúde pública acarretou mudanças na forma de atender clientes e interagir com colegas, exigindo a rápida transição da modalidade presencial para a modalidade remota, em muitos casos. O presente estudo sistematizou conhecimentos sobre terapia on-line com casais e famílias, trazendo considerações para a prática e a formação profissional diante da pandemia. Por meio de revisão narrativa da literatura, foram sumarizados resultados de estudos empíricos, bem como recomendações sobre aspectos técnicos, éticos e formativos. Destacaram-se, em particular, potencialidades e desafios para a utilização de tecnologias da informação e da comunicação na prática clínica com casais e famílias, incluindo indicações e contraindicações, recursos mais apropriados e seguros, relação terapêutica on-line, capacitação e supervisão. Entende-se que a Covid-19 representa uma oportunidade para revisão e reflexão da prática clínica e da formação profissional de terapeutas de casal e família no Brasil, país em que as intervenções on-line permaneciam relativamente pouco exploradas até a pandemia.
\end{abstract}

Palavras-Chave: Terapia On-line, Terapia Familiar, Terapia de Casais, Formação Profissional, Supervisão Clínica.

\section{Online Therapy with Couples and Families: Practice and Training in the Covid-19 Pandemic}

\begin{abstract}
The Covid-19 pandemic has boosted the already emerging process of virtual connections. For couple and family therapists, this major public health crisis has led to changes in the way of treating clients and interacting with colleagues, requiring rapid transition from faceto-face to remote mode, in many cases. This study systematized knowledge about online therapy with couples and families, bringing considerations for practice and professional training in the face of the pandemic. By conducting a narrative literature review, we summarized results from empirical studies, as well as recommendations on technical, ethical, and formative aspects. Specifically, we highlighted both the potential and the challenges of using information and communication technologies in clinical practice with couples and families, including indications and contraindications, more appropriate and safer resources, online therapeutic relationship, training, and supervision. Covid-19 represents an opportunity for reviewing and analyzing the clinical practice and professional training of couples and family therapists in Brazil, a country in which online interventions remained relatively little explored until the pandemic.
\end{abstract}

Keywords: Online Therapy, Family Therapy, Couples Therapy, Professional Education, Clinical Supervision. 


\title{
Terapia de Parejas y Familias On line: Práctica y Formación en la Pandemia de Covid-19
}

\begin{abstract}
Resumen: La pandemia de Covid-19 potenció el proceso ya emergente de conexiones virtuales. Para terapeutas de parejas y familias, esa grave emergencia de salud pública acarreó cambios en la forma de atender a clientes e interactuar con colegas, exigiendo la rápida transición de la modalidad presencial para la modalidad remota, en muchos casos. El presente estudio sistematizó conocimientos sobre terapia de parejas y familias on line, trayendo consideraciones para la práctica y la formación profesional en la pandemia. Por medio de revisión narrativa de la literatura, fueron obtenidos resultados de estudios empíricos, así como recomendaciones sobre aspectos técnicos, éticos y formativos. Se destacaron, en particular, potencialidades y desafíos para usar tecnologías de la información y de la comunicación en la práctica clínica con parejas y familias, incluyendo indicaciones y contraindicaciones, recursos más apropiados y seguros, relación terapéutica on line, capacitación y supervisión. Se entiende que el Covid-19 representa una oportunidad para la revisión y reflexión de la práctica clínica y de la formación profesional de terapeutas de parejas y familias en Brasil, donde las intervenciones on line permanecían relativamente poco exploradas hasta la pandemia.
\end{abstract}

Palabras clave: Terapia On line, Terapia Familiar, Terapia de Parejas, Formación Profesional, Supervisión Clínica.

A doença causada pelo novo coronavírus (Covid-19) tem afetado o mundo de diferentes formas desde sua primeira notificação, em dezembro de 2019, na China (Inchausti, MacBeth, HassonOhayon, \& Dimaggio, 2020). Na terceira semana de agosto de 2020 (quando a redação do presente artigo foi concluída), havia o registro global de aproximadamente 22 milhões de casos de infecção e 775 mil óbitos por Covid-19 (World Health Organization, 2020). Nesse mesmo período, o Brasil registrava 3,3 milhões de casos de infecção e 109 mil óbitos, atrás apenas dos Estados Unidos. Visto que a Covid-19 é uma doença altamente contagiosa, para a qual tratamentos e vacinas ainda estão em fase experimental, as principais formas de prevenção do contágio envolvem distanciamento social, higienização das mãos e uso de máscaras (Gritti, Salvati, Russo, \& Catone, 2020). Nesse sentido, afora as repercussões diretas, que podem incluir adoecimento, hospitalização e morte, as repercussões indiretas da Covid-19, desencadeadas sobretudo em função das medidas adotadas para conter a pandemia, também têm afetado a saúde mental e as relações interpessoais, com destaque às relações familiares (Prime, Wade, \& Browne, 2020; Schmidt, Crepaldi, Bolze, Neiva-Silva, \& Demenech, 2020).
Em decorrência do distanciamento social, por exemplo, a rotina das famílias sofreu mudanças significativas em um curto espaço de tempo (Prime et al., 2020; Silva, Lordello, Schmidt, \& Mieto, 2020). Escolas foram temporariamente fechadas em todo o mundo, sendo que muitas crianças e adolescentes não tiveram as atividades de ensino presencial substituídas pela modalidade remota (United Nations Children's Fund, 2020). Os adultos, por outro lado, podem ter experienciado transformações nas atividades laborais, tais como a necessidade de realizar teletrabalho, a redução da carga horária e, por conseguinte, da renda, ou ainda a perda do emprego (Oliveira, Abranches, \& Lana, 2020). Para alguns trabalhadores, em particular, houve o aumento da carga horária ou a necessidade de se manterem fisicamente afastados de seus familiares, como é o caso dos profissionais da saúde que atuam na "linha de frente", em contato direto com pessoas infectadas pelo novo coronavírus (Schmidt et al., 2020).

Os idosos, população especialmente vulnerável a complicações pela Covid-19, também foram desencorajados a manter contatos face a face com outras pessoas (Prime et al., 2020; Schmidt et al., 2020). Ademais, mesmo aqueles idosos que até a pandemia realizavam suas atividades cotidianas de modo independente 
passaram a necessitar de apoio, para evitar a infecção (Coyne et al., 2020). Essas mudanças na rotina das famílias têm sobrecarregado notadamente as mulheres, que tradicionalmente são as principais responsáveis pelos cuidados a crianças, adolescentes, idosos e familiares que adoecem, além de ainda realizarem grande parte das tarefas domésticas (Silva et al., 2020).

A necessidade de convívio por períodos prolongados no domicílio pode tanto fortalecer os laços afetivos e a resiliência familiar quanto aumentar o nível de estresse e os conflitos entre os membros da família (Lebow, 2020; Prime et al., 2020). Portanto, lidar de forma construtiva com as adversidades desencadeadas ou intensificadas a partir da pandemia, incluindo os conflitos pelo compartilhamento do espaço e das tarefas domésticas, pode favorecer recursos familiares, por meio da busca de soluções cooperativas para os desafios enfrentados (Gritti, 2020). Por outro lado, algumas famílias tendem a se tornar mais vulneráveis nesse contexto de intensificação da convivência (Lebow, 2020). Isso pode ocorrer notadamente com famílias cujos laços afetivos já se mostravam fragilizados (Gritti, 2020) e que, em decorrência da pandemia, passaram a experienciar uma sobreposição de estressores, tais como dificuldades financeiras e desarticulação das redes de apoio (Marques, Moraes, Hasselmann, Deslandes, \& Reichenheim, 2020). Nesse sentido, os conflitos conjugais, parentais e coparentais têm se destacado (Prime et al., 2020), o que se associa a um maior risco de violência familiar (Gritti, 2020; Marques et al., 2020).

A Covid-19 também potencializou o processo já emergente de conexões virtuais, de maneira que os contatos com as pessoas que não residem no domicílio familiar passaram a ocorrer predominantemente via internet ou por telefone, o que traz novas possibilidades de conexões e, por outro lado, o desafio da perda daquelas previamente estabelecidas (Lebow, 2020). Assim, embora as distâncias geográficas pareçam um fator menos preponderante para manter as relações interpessoais no atual cenário (Borcsa \& Pomini, 2018; Hertlein, Blumer, \& Smith, 2014), é importante considerar os impactos da restrição das interações face a face nos diferentes estágios do ciclo de vida. Crianças e adultos, por exemplo, perderam a socialização com referências do contexto ampliado, representado pela escola e pelo ambiente de trabalho, respectivamente, o que requer reorganização interna e reestruturação do ambiente doméstico (Linhares \& Enumo, 2020).
Além disso, os idosos tendem a ser desproporcionalmente afetados nesse cenário, pois, além das restrições em atividades e interações cotidianas, eles costumam apresentar dificuldades para utilizar recursos tecnológicos (Schmidt et al., 2020).

A pandemia também acarretou mudanças importantes na forma como psicólogos e outros profissionais da saúde mental prestam atendimento a seus clientes e interagem com colegas, exigindo a rápida transição da modalidade presencial para a modalidade remota, em muitos casos (Gritti, 2020; Matheson, Bohon, \& Lock, 2020; Wade et al., 2020). Em atenção a essa demanda emergente, no Brasil, o Conselho Federal de Psicologia (CFP) regulamentou a expansão dos serviços psicológicos prestados por meio das tecnologias da informação e da comunicação (TICs) durante a pandemia (Conselho Federal de Psicologia, 2020a). Não obstante, questões complexas sobre atendimentos remotos têm sido levantadas, sobretudo por terapeutas de casal e família, os quais, até então, vinham realizando seu trabalho quase exclusivamente na modalidade presencial (Lebow, 2020; Pickens, Morris, \& Johnson, 2020). Essas questões incluem aspectos técnicos e éticos, com destaque a como engajar os membros da família via videoconferência, evitar distrações no ambiente doméstico, adaptar intervenções que envolvam crianças pequenas e garantir a privacidade dos membros da família que estão em atendimento frente àqueles que não estão (Lebow, 2020; Wade et al., 2020).

Nesse contexto, terapeutas de casal e família têm referido preocupações quanto à relação terapêutica, à efetividade dos atendimentos remotos bem como aos recursos mais apropriados e seguros para realizá-los (Lebow, 2020; Wade et al., 2020). As fragilidades na formação em terapia on-line com casais e famílias se associam também a dúvidas quanto a indicações e contraindicações dessa modalidade, inclusive entre profissionais com experiência em terapia on-line individual (Wrape \& McGinn, 2019). Assim, capacitações profissionais e supervisões têm se mostrado relevantes para a qualificação da terapia on-line com casais e famílias (Pickens et al., 2020), podendo ser realizadas por meio das TICs (Borcsa \& Pomini, 2018; Pennington, Patton, Ray, \& Katafiasz, 2017).

Diante do exposto, o objetivo do presente estudo é sistematizar conhecimentos sobre a terapia on-line com casais e famílias, trazendo considerações para a prática e a formação profissional no contexto da 
pandemia de Covid-19. Foi realizada uma revisão narrativa da literatura, modalidade que se mostra apropriada para sumarizar conhecimentos a respeito de temas relativamente pouco explorados, buscando oferecer subsídios para intervenções (Ferrari, 2015). Nesse sentido, foram sumarizados resultados de estudos empíricos, além de recomendações técnicas e éticas disponibilizadas por pesquisadores e associações profissionais de diferentes países. Ademais, com base na experiência profissional e de pesquisa das autoras do presente estudo em dinâmicas relacionais e intervenções conjugais e familiares, bem como terapia on-line, apresentamos e discutimos aqui as potencialidades e os desafios para a utilização das TICs na prática clínica com casais e famílias no contexto brasileiro.

\section{Terapia on-line: história, pesquisa e prática}

A terapia on-line vem sendo praticada há mais de duas décadas em diferentes partes do mundo (Barak, Hen, Boniel-Nissim, \& Shapira, 2008; Grohol, 2004). Essa modalidade terapêutica deriva dos grupos de apoio na web dos anos de 1970, dos serviços universitários norte-americanos de atendimento psicológico on-line gratuito dos anos de 1980, da terapia por telefone e da videoconferência psiquiátrica (Simpson, 2003; Skinner \& Zack, 2004). Suas influências remotas têm origem nos programas de computador que substituíram os terapeutas humanos na década de 1960 (Grohol, 2004) e na prática da correspondência terapêutica, iniciada por Freud (Skinner \& Zack, 2004). A terapia on-line começou a crescer em meados da década de 1990, quando a internet experimentou uma expansão significativa, com centenas de milhares de novos usuários todos os meses. Não é de surpreender que alguns desses indivíduos estivessem interessados em obter serviços psicológicos via web (Grohol, 2004). No Brasil, em 1999, o Núcleo de Pesquisa em Psicologia e Informática da Pontifícia Universidade Católica de São Paulo (PUC-SP) começou a receber via e-mail uma demanda por apoio psicológico na rede e passou a responder à mesma, oferecendo regularmente orientação psicológica via e-mail (Fortim \& Cosentino, 2007). Essa é a experiência pioneira em atendimento psicológico on-line de que se tem notícia no país.

A prática da terapia on-line se dá por meio de videoconferência, audioconferência ou texto (chat, SMS e e-mail), de forma síncrona (interação simultânea) e/ou assíncrona (interação não simultânea). Essa modalidade terapêutica oferece vantagens como disponibilidade, conveniência e redução de estigma (Cartreine, Ahern, \& Locke, 2010), ampliando o acesso a tratamentos psicológicos (Proudfoot et al., 2011). Em seu início, a terapia on-line recebeu grande oposição daqueles que rejeitavam a ideia de que as pessoas poderiam estabelecer um relacionamento real mediado pelo computador (Skinner \& Zack, 2004). Muitos terapeutas estão preocupados em ser menos capazes de comunicar empatia e construir uma aliança terapêutica na rede (Roesler, 2017). Entretanto, estudos vêm mostrando que a relação terapêutica na terapia on-line, nas mais distintas modalidades, síncronas ou assíncronas, pode equivaler à da terapia presencial (Berger, 2017; Richards \& Vigano, 2013; Simpson \& Reid, 2014).

Elementos da relação terapêutica como confiança (Fletcher-Tomenius \& Vossler, 2009), conexão (Bouchard et al., 2004) e empatia (Himle et al., 2006) desenvolvem-se também on-line. $\mathrm{O}$ sentimento de clientes e terapeutas de estarem juntos, embora ligados em rede, é conhecido como telepresença, a percepção ilusória de não-mediação (Lombard \& Ditton, 1997). A telepresença é fator central no desenvolvimento de relações terapêuticas profundas (Anthony, 2000; Himle et al., 2006) e é comum em atendimentos via videoconferência, que se aproximam da terapia presencial devido ao recurso de áudio e vídeo (Berger, 2017). Não é à toa que a maior parte dos terapeutas norte-americanos prefere a videoconferência como meio para prestar seus serviços psicológicos on-line (Glueckauf et al., 2018).

Muitos terapeutas acreditam incorretamente que as terapias on-line são menos efetivas do que as sessões realizadas presencialmente (Bekés \& Doorn, 2020). Machado et al. (2016) conduziram uma revisão sistemática sobre terapia individual on-line e observaram que essa, quando comparada à terapia presencial, mostra efeitos similares. Constataram também que as diversas formas de comunicação entre cliente e terapeuta permitem o estabelecimento de diferentes relações terapêuticas e possibilidades clínicas. Os autores concluíram que a terapia individual on-line, síncrona e assíncrona, caracteriza-se como uma forma de tratamento viável.

No Brasil, já há resultados positivos de intervenções psicológicas on-line para tratamentos individuais. Pieta (2014), utilizando o Inventário de Aliança 
Terapêutica (IAT) e o questionário de resultados terapêuticos Outcome Questionnaire (OQ45), verificou uma pontuação alta na relação entre clientes e psicoterapeutas em psicoterapia psicanalítica via videoconferência de 12 semanas de duração, bem como diminuição significativa dos sintomas dos clientes. Rodrigues (2014), também utilizando o IAT, observou que a aliança terapêutica dos clientes que receberam psicoterapia breve focal via videoconferência manteve-se ao longo do processo psicoterápico e mostrou-se semelhante à presencial. Faria (2019) ofereceu Gestalt-terapia breve via chat e videoconferência e considerou que o vínculo terapêutico que se estabeleceu foi satisfatório.

Não obstante, apesar dos resultados encorajadores em termos de eficácia e efetividade da terapia on-line para tratamentos individuais considerando diferentes abordagens terapêuticas, sabe-se relativamente pouco sobre o uso das TICs por terapeutas de casal e família (Caldwell, Bischoff, Derigg-Palumbo, \& Liebert, 2017; Hertlein et al. 2014; Lebow, 2020). Ainda que conduzidas junto a pequenas amostras, pesquisas sobre terapia on-line com casais e famílias têm sugerido os benefícios dessa modalidade de intervenção (Borcsa \& Pomini, 2018; Wrape \& McGinn, 2019), conforme será abordado na seção a seguir.

\section{Aspectos técnicos e éticos da terapia on-line com casais e famílias}

Anteriormente à pandemia de Covid-19, as TICs vinham sendo utilizadas por terapeutas de casal e família sobretudo para primeiros contatos, comunicações breves, encaminhamentos a demandas emergentes entre sessões, envio de materiais psicoeducativos ou lembretes de horários agendados, usando recursos como telefonemas, e-mails e mensagens de texto (Caldwell et al., 2017; Hertlein et al. 2014; Pennington et al., 2017). Atendimentos via videoconferência, por outro lado, vinham sendo relativamente pouco utilizados (Lebow, 2020), apesar do crescente corpo de evidências apoiando-os como um modo eficaz para oferecer serviços terapêuticos (Pickens et al., 2020).

Alguns clientes podem se mostrar particularmente abertos à terapia on-line, pela conveniência de realizar as sessões da própria casa, evitando deslocamentos, ou pela possibilidade de conectar membros da família fisicamente distantes, como casais que residem em cidades diferentes (Borcsa \& Pomini, 2018; Wrape \& McGinn, 2019). Além disso, o estigma referente a problemas relacionais (p. ex., conflitos familiares, traição conjugal, disfunções sexuais), que consiste em uma barreira à busca por atendimento presencial, pode ser mais facilmente superado na terapia on-line (Roddy, Rothman, Cicila, \& Doss, 2019). Ainda, para pessoas que moram em regiões com escassez de oferta de terapia presencial, a terapia on-line se mostra especialmente importante (Inchausti et al., 2020; Pickens et al., 2020; Wade et al., 2020). Sessões remotas podem ser intercaladas com sessões face a face, caso isso seja avaliado como uma opção viável no plano terapêutico do casal ou da família (Wrape \& McGinn, 2019).

Contudo, terapeutas de casal e família frequentemente se sentem pouco preparados para realizar terapia on-line. Em estudo conduzido nos Estados Unidos, do qual participaram 169 terapeutas, supervisores e estudantes de terapia de casal e família, constatou-se que a maioria deles se sentiria muito desconfortável ao tratar seus clientes exclusivamente por meio de atendimentos on-line (Hertlein et al. 2014). Para os autores, tal desconforto poderia ser explicado pela percepção dos profissionais de que os atendimentos on-line interferem na aliança terapêutica, prejudicam manifestações de empatia e sensibilidade, bem como dificultam o controle do setting, pois não é possível manejar uma reação adversa à distância da mesma forma que se faz presencialmente. Aspectos éticos também podem trazer preocupações aos profissionais, como revelou um outro estudo conduzido nos Estados Unidos, do qual participaram 226 terapeutas, supervisores e estudantes de terapia de casal e família (Hertlein, Blumer, \& Mihaloliakos, 2015). Nesse estudo, preocupações sobre a confidencialidade e a privacidade nos atendimentos foram referidas pela maioria dos participantes, incluindo a segurança no local onde os clientes se encontravam (p. ex., quem mais estaria naquele ambiente?) e a segurança on-line (p. ex., qual a garantia de conexão segura, impedindo o acesso de outras pessoas aos atendimentos remotos?).

Não obstante, no contexto da pandemia de Covid-19, observou-se uma ampla e rápida transição da oferta de atendimentos presenciais para aqueles na modalidade remota (Gritti, 2020; Matheson et al., 2020). Em linhas gerais, essa transição foi motivada pelas medidas de distanciamento social. Entretanto, mesmo nos casos em que é possível realizar atendimentos presenciais em condições adequadas de proteção contra o novo coronavírus - em ambientes ventilados, 
com manutenção de distância física entre as pessoas e uso de máscaras, por exemplo - há quem opte pela modalidade remota, por necessidade ou conveniência (MacMullin, Jerry, \& Cook, 2020). Portanto, destaca-se a relevância da qualificação da terapia on-line, sobretudo ao considerar que o tempo de duração da pandemia é imprevisível (Schmidt et al., 2020) e que, mesmo ao final dela, há chances de essa modalidade se tornar mais disseminada e vir a ser integrada em definitivo à prática de muitos terapeutas de casal e família, os quais precisarão reconhecer suas potencialidades e desafios (MacMullin et al., 2020). Nesse sentido, é possível traçar um paralelo entre os esforços atuais para adaptar o campo às demandas da era digital e da pandemia e os esforços dos pioneiros da terapia de casal e família para mudar o foco do tratamento individual para o trabalho com sistemas humanos, visto que preocupações sobre aspectos técnicos e éticos também surgiram naquele momento (Pickens et al., 2020).

Dado que aspectos técnicos e éticos estão inexoravelmente entrelaçados (Wrape \& McGinn, 2019), no presente artigo eles são tratados de forma interrelacionada e não mutuamente exclusiva. Preocupações sobre confidencialidade e privacidade ocorrem nos atendimentos remotos a casais e famílias, mas também nos atendimentos individuais (Hertlein et al., 2015; Lustgarten, Garrison, Sinnard, \& Flynn, 2020). Nesse sentido, um primeiro ponto a se considerar diz respeito à infraestrutura, sendo necessário garantir a criptografia de ponta a ponta nas comunicações (como videoconferências, audioconferências e mensagens de texto), bem como a segurança e a confiabilidade da rede de internet (i.e., conexão segura, em vez de Wi-Fipúblico/gratuito) e do software utilizado (i.e., plataforma em que ocorrem os atendimentos remotos, tais como Zoom ou Skype) (Caldwell et al., 2017; Lustgarten et al., 2020). Os equipamentos (p. ex., computadores e câmeras) precisam ser funcionais e seguros. Em caso de falha, equipamentos de reserva devem estar disponíveis para que o profissional realize substituição imediata. Recomenda-se verificar se o antivírus está atualizado. Além disso, senhas devem ser trocadas com frequência (Caldwell et al., 2017).

Uma das ferramentas mais importantes à disposição dos terapeutas de casal e família quando se trata de confidencialidade e privacidade é o consentimento informado. Portanto, no contrato dos serviços a serem prestados, além da menção aos potenciais riscos e benefícios da terapia on-line
(Caldwell et al., 2017), é essencial declarar que, embora a confidencialidade e o sigilo sejam responsabilidades do psicólogo (Conselho Federal de Psicologia, 2018), os clientes são corresponsáveis por proteger sua própria privacidade, discutindo com eles de que forma isso pode ser feito (Wrape \& McGinn, 2019). Tais questões devem ser retomadas ao longo do processo terapêutico. Sugere-se também que o consentimento informado explicite $o$ plano para o caso de falhas tecnológicas. Isso inclui como proceder para reestabelecer a conexão, recursos alternativos (p. ex., audioconferência, caso haja problemas com a videoconferência), além de orientações sobre os valores referentes a sessões em que ocorrerem falhas tecnológicas (Caldwell et al., 2017).

Outro ponto a ser considerado é a segurança dos membros do casal ou da família. Nesse sentido, há contraindicações para atendimentos na modalidade remota nos casos de violência por parceiro íntimo, alto risco de suicídio e abuso de substâncias não-tratado (Borcsa \& Pomini, 2018; Hertlein et al. 2015). Para avaliar a segurança, sugere-se inicialmente a realização de entrevistas individuais com cada um dos membros do casal ou da família, o que também permite examinar o status clínico e cognitivo dos clientes (American Psychological Association, 2020), analisar a viabilidade da terapia on-line e planejar o tratamento (Wrape \& McGinn, 2019). Nas entrevistas individuais, deve-se solicitar que os clientes utilizem fones de ouvido, enquanto o profissional faz perguntas sobre temas sensíveis, cujas respostas são "sim" ou "não". Isso assegura a confidencialidade, ainda que outra pessoa esteja escutando a conversa. Essa mesma estratégia deve ser usada com todos os membros do casal ou da família para manter a equidade (Wrape \& McGinn, 2019). Cabe salientar que, na avaliação inicial, o terapeuta deve considerar o tipo e a severidade dos sintomas, a natureza do tratamento a ser oferecido, a adequação da infraestrutura que o casal ou a família dispõe para realizar as sessões, bem como a habilidade deles em utilizar as TICs. Com base nesses aspectos, é possível analisar se a terapia on-line é a modalidade mais adequada para atender às demandas apresentadas pelo casal ou pela família (Caldwell et al., 2017).

Nos atendimentos remotos, deve-se estar atento a casais e famílias que demonstram a tendência a rápidas escaladas na severidade dos conflitos, o que costuma demandar redirecionamentos por parte do terapeuta (Matheson et al., 2020; Wrape \& 
McGinn, 2019). Nesses casos, o manejo pode envolver a solicitação de que um dos clientes deixe o cômodo onde a sessão está sendo realizada, fazendo uma pausa para regulação de emoções, com incentivo para retorno após alguns minutos (Time Out). Isso deve ocorrer somente quando a segurança não for uma preocupação. É possível que o cliente deixe o cômodo, mas continue próximo, ouvindo as conversações entre o terapeuta e o cônjuge ou os demais familiares em sua ausência (Matheson et al., 2020). Pode-se estabelecer com os clientes a realização de um sinal com as mãos (p. ex., “T”), quando alguém sentir a necessidade de interromper a sessão temporariamente. Visto que o controle do setting é reduzido em comparação à terapia presencial, atenção especial deve ser dada ao plano de gerenciamento de crises, o que inclui a possibilidade de verificar a localização do casal ou da família e acionar serviços ou profissionais da rede local, se necessário. Recomenda-se que aspectos referentes ao gerenciamento de crises e aos encaminhamentos sejam discutidos no contrato e constem no consentimento informado (Wrape \& McGinn, 2019).

A participação de múltiplos clientes em uma mesma sessão é um desafio inerente à prática dos terapeutas de casal e família. Esse desafio, entretanto, é potencializado na terapia on-line (Lebow, 2020), a qual demanda a organização de áudio, iluminação e vídeo, de modo a permitir o contato visual com cada indivíduo em frente à câmera (Wrape \& McGinn, 2019). O setting pode variar, tendo em vista que os membros do casal ou da família podem estar em uma ou mais telas, no mesmo ambiente ou não (Borcsa \& Pomini, 2018). Se o casal ou a família utilizar uma única tela, o terapeuta pode solicitar que se posicionem em círculo ou semicírculo, ao invés de linha reta, no sentido de promover uma melhor comunicação entre os membros.

Adicionalmente, embora realizar a sessão no ambiente doméstico possa ser considerado mais confortável por muitos clientes, há casais e famílias que apresentam dificuldades para conseguir um espaço com privacidade, livre de interrupções, ou mesmo para organizar os cuidados dos filhos durante a sessão. Recomenda-se que o terapeuta discuta esses aspectos com os clientes de modo colaborativo e sensível. Deve-se abordar também a importância de evitar distrações no ambiente doméstico no momento dos atendimentos. Isso pode incluir acordos sobre desligar a televisão ou outros dispositivos eletrônicos, não realizar refeições e manter os animais de estimação em um cômodo à parte (Matheson et al., 2020).

Embora a comunicação não-verbal dos clientes possa ser observada nos atendimentos remotos, o profissional tende a apresentar maiores dificuldades para compreendê-la e utilizá-la como recurso terapêutico (Inchausti et al., 2020; Wrape \& McGinn, 2019). Similarmente, técnicas não-verbais, como as propostas pelo modelo estrutural sistêmico para sinalizar alianças temporárias (p. ex., mover uma cadeira para se aproximar de um membro da família) não são facilmente adaptáveis à terapia on-line. A tecnologia também pode criar interrupções, o que inclui perda do sinal da internet e término da bateria do equipamento. Nesse sentido, os silêncios, comumente impactantes nos atendimentos presenciais (Matheson et al., 2020), podem ser confundidos com falhas tecnológicas na terapia on-line (Pieta, 2014).

Em conjunto, esses aspectos demandam que o terapeuta trabalhe para garantir a participação ativa de todos os membros do casal ou da família nos atendimentos remotos, o que inclui a menção frequente aos nomes e o direcionamento de perguntas específicas para cada pessoa nos questionamentos circulares, por exemplo (Wrape \& McGinn, 2019). Acenos sutis com a cabeça, recorrentemente usados nos atendimentos presenciais, tendem a ser menos efetivos na terapia on-line. Para lidar com essas limitações dos atendimentos remotos, o terapeuta pode alterar a entonação ou a cadência da voz, demonstrar modificações mais acentuadas nas suas expressões faciais, bem como se inclinar na direção de alguma pessoa em específico na tela do computador, de forma a se comunicar mais efetivamente com os membros do casal ou da família (Matheson et al., 2020). Nesse contexto, recomenda-se o aumento da frequência de perguntas como "o que você está sentindo?" e "o que lhe ocorre agora?", o que tende a favorecer a expressão verbal das experiências emocionais, permitindo aos clientes interagirem de maneira diferente entre si e com suas emoções (Wrape \& McGinn, 2019).

Em linhas gerais, o ambiente digital requer que o terapeuta adote alternativas criativas às técnicas frequentemente utilizadas nos atendimentos presenciais, sem desconsiderar aspectos éticos (Inchausti et al., 2020). No entanto, essa tarefa 
é desafiadora, especialmente porque a terapia on-line com casais e famílias vinha sendo pouco explorada em termos de formação profissional (Hertlein et al. 2015; Matheson et al., 2020), o que será abordado na seção a seguir.

\section{Formação para oferta de terapia on-line com casais e famílias}

Em uma situação de emergência, como a pandemia de Covid-19, deve-se buscar o equilíbrio entre as necessidades de capacitação dos profissionais que atendem casais e famílias e as necessidades daqueles que demandam esse serviço (McCord et al., 2020). Estudos internacionais relatam que a maior parte dos terapeutas de casal e família declaram não ter aprendido sobre o uso das TICs na prática clínica, o que sugere que uma parcela expressiva dos profissionais da área não foi capacitada para oferecer terapia on-line (Blumer, Hertlein, \& VandenBosch, 2015; Borcsa \& Pomini, 2018; Hertlein et al., 2014).

Programas que enfocam a formação de terapeutas para a oferta de terapia on-line em outras áreas da Psicologia oferecem alguns direcionamentos que poderiam ser seguidos pelos programas destinados aos terapeutas de casal e família. No Texas $A \& M$ Telebehavioral Care Program, nos Estados Unidos, os terapeutas em formação conduzem seus atendimentos a partir do próprio serviço, em um ambiente em que se garante a adequabilidade das condições técnicas para a oferta da terapia on-line, bem como o acesso a supervisores e técnicos do serviço, que podem intervir em caso de necessidade (McCord et al., 2020). Diante da pandemia de Covid-19, foi necessário implementar um novo protocolo. O programa passou, então, a disponibilizar: a) recursos que permitissem centralizar as ligações entre terapeutas e clientes, de forma que estes não tivessem acesso aos números pessoais daqueles; b) programas de videoconferência que permitissem ao supervisor o acompanhamento da sessão em casos de emergência; e, c) laptops, fones de ouvido e demais tecnologias necessárias ao atendimento. O programa também garantiu a supervisão remota semanal em grupo aos terapeutas em formação, além de encontros virtuais individuais com o supervisor que permitissem trabalhar questões pessoais que estivessem sendo vivenciadas pelos terapeutas em função da pandemia.

Acredita-se, no entanto, que muitas instituições de ensino (como universidades ou centros de especialização/formação) no Brasil enfrentariam dificuldades para oferecer esses mesmos recursos a seus estudantes, inclusive porque a prestação de serviços psicológicos por meio das TICs foi regulamentada recentemente no país (Conselho Federal de Psicologia, 2012, 2018). Uma das principais dificuldades a ser superada seria o desconhecimento do tema por professores e formadores. Estudo conduzido com professores e pesquisadores de Psicologia Clínica do Sul do Brasil sugeriu pouco conhecimento sobre o tema, dúvidas acerca da base legal para a terapia on-line, bem como desconfiança sobre os benefícios dessa prática (Ribeiro, 2005). Embora se trate de um estudo antigo, a escassez de estudos nacionais recentes sobre o tema sugere que a situação possa se manter na atualidade.

Outro importante desafio à capacitação em terapia on-line é a elevada desigualdade social no Brasil, o que tende a afetar a formação de estudantes com dificuldades de acesso à internet ou que não disponham de ferramentas tecnológicas (além do que, evidentemente, também pode excluir dos atendimentos ofertados pelas instituições de ensino segmentos vulneráveis da população, devido a seu baixo acesso às TICs). Buscando atender essa e outras demandas referentes a atividades formativas de caráter prático no contexto da Covid-19, o CFP e a Associação Brasileira de Ensino de Psicologia (ABEP), juntamente aos Conselhos Regionais de Psicologia e aos Núcleos da ABEP, promoveram os Seminários Regionais e Nacional de Formação em Psicologia em tempos de pandemia. Esses eventos ocorreram entre junho e julho de 2020, com participação de coordenadores, docentes, supervisores e estudantes de Psicologia. Como resultado desse processo de discussão, em agosto de 2020 foi publicado um material com recomendações para estágios remotos durante a pandemia de Covid-19 (Conselho Federal de Psicologia, 2020b).

Os estágios remotos têm caráter excepcional, de modo que as atividades práticas presenciais devem ser retomadas assim que a crise sanitária for superada (Conselho Federal de Psicologia, 2020b). No que diz respeito aos estágios remotos clínicos, em particular, a indicação é de que sejam realizados nas dependências dos serviços-escola, no sentido de favorecer a garantia de privacidade, sigilo e confiabilidade das atividades práticas desenvolvidas pelos estagiários. Ademais, tanto estagiários quanto supervisores devem ter conhecimento sobre as TICs que serão utilizadas, 
bem como acesso institucionalmente garantido a essas ferramentas. As instituições de ensino também devem assegurar condições de biossegurança (p. ex., equipamentos de proteção individual, marcadores de medidas de distanciamento no chão, ventilação natural nos ambientes, higienização das salas entre os atendimentos e planejamento dos horários de entrada e saída de estudantes, supervisores e docentes). Além da continuidade dos atendimentos terapêuticos iniciados na modalidade presencial, o CFP (2020b) sugere a realização de plantões psicológicos (com acolhimentos e encaminhamentos), bem como reuniões de equipe nos estágios remotos clínicos. Destaca-se, entretanto, que o material produzido pelo CFP (2020b) não apresenta considerações específicas para o estágio em terapia de casal e família, o que revela a necessidade de serem discutidas as particularidades dessa prática nas instituições de ensino durante a pandemia de Covid-19.

Os cursos de especialização e formação de terapeutas de casal e família também devem se mostrar atentos a esse debate e pensar em como inserir esse tema na formação de seus estudantes, já que hoje esse não é um tópico obrigatoriamente abordado pelos cursos, segundo a Associação Brasileira de Terapia Familiar (ABRATEF, 2018). Dentre os principais temas a serem abordados na capacitação dos terapeutas de casal e família para a prática da terapia on-line, estão: a) orientações práticas sobre como conduzir atendimentos on-line; b) questões éticas e legais; c) cuidados a serem adotados para garantir o sigilo e a confidencialidade dos clientes; d) medidas para garantir a segurança de terapeutas e clientes nesse contexto; e, e) evidências de pesquisas sobre terapia on-line com casais e famílias (Blumer et al., 2015). Conforme assinalaram McCord et al. (2020), a Covid-19 destacou a importância da capacitação dos profissionais para a oferta da terapia on-line, e essa é uma questão que deve seguir sendo pensada mesmo após o fim da pandemia.

\section{Supervisão on-line a terapeutas de casal e família}

A supervisão é um dos principais instrumentos na formação de terapeutas, contribuindo tanto para a qualidade dos atendimentos prestados como para a satisfação profissional (Kanz, 2001; Twist, Hertlein, \& Haider, 2016). Por meio dela, desenvolve-se a identidade profissional, adquirem-se e aprimoram-se habilidades e competências clínicas (Perry, 2012).
Na formação em Psicologia, a supervisão é obrigatória para estudantes de graduação (Conselho Federal de Psicologia, 2013). É também utilizada por profissionais já formados que desejam aprimorar sua prática ou que cursam especialização/formação na área clínica. A ABRATEF (2018), por exemplo, dispõe que os cursos de formação em terapia de casal e família prevejam uma carga horária mínima de 100 horas de atendimento clínico supervisionado.

A supervisão on-line inclui a troca de informações entre supervisores e terapeutas por meio de telefone, e-mail, aplicativos de mensagem instantânea ou videoconferência. Pode ser adaptada para atender aos objetivos de diferentes modalidades de supervisão utilizadas na área de terapia de casal e família. $\mathrm{Na}$ supervisão relatada on-line, o terapeuta relata os atendimentos realizados e os discute com o supervisor, em um formato semelhante àquele adotado nas supervisões relatadas presenciais (Rousmaniere, Abbass, \& Frederickson, 2014). Na supervisão ao vivo on-line, o supervisor acompanha - por meio de recursos de videoconferência - $o$ atendimento enquanto ele é realizado e envia orientações ao(s) terapeuta(s). As mensagens do supervisor são transmitidas por meio de um fone de ouvido, que o terapeuta utiliza ao longo da sessão, ou de mensagens de texto que são exibidas em uma tela situada ao lado ou atrás dos clientes (Bacigalupe, 2010; Rousmaniere et al., 2014). Embora iniciativas de supervisão on-line no campo da terapia de casal e família tenham sido propostas já na década de 1990, ainda são escassos os estudos sobre seu uso na supervisão de terapeutas de casal e família (Tarlow, McCord, Nelon, \& Bernhard, 2020).

No Brasil, as supervisões on-line foram autorizadas em 2012, para a supervisão eventual de psicólogos e para a realização de supervisões complementares à sua formação presencial profissional (Conselho Federal de Psicologia, 2012). A partir de 2018, a supervisão on-line passou a ser autorizada nos mais diversos contextos, desde que o supervisor tenha registro no CRP e cadastro no e-Psi (Conselho Federal de Psicologia, 2018, 2020b).

A supervisão on-line tem se mostrado útil ao permitir o acesso à supervisão qualificada a terapeutas que atuam em regiões afastadas ou cidades pequenas, bem como em regiões em que haja poucos profissionais que atuem na abordagem escolhida ou que tenham experiência em temáticas específicas (Bacigalupe, 2010; Borcsa \& Pomini, 2018; Conn, Roberts, \& Powell, 2009; Lenz, Oliver, \& Nelson, 2011; Twist et al., 2016). 
A pandemia de Covid-19 tem reforçado a demanda por esse tipo de supervisão, visto que os terapeutas que começaram a oferecer terapia on-line também passaram a buscar supervisão nesse formato (Tarlow et al., 2020). Nesse contexto, a supervisão remota representa uma importante estratégia para garantir o atendimento às necessidades dos clientes e o cuidado com a saúde mental dos terapeutas, que se encontram em risco de burnoute trauma secundário devido ao excesso de responsabilidades profissionais, ao impacto do estado de pandemia eà pouca experiência em lidar com situações de emergência e desastre (Tarlow et al., 2020). É necessário, porém, que a adesão à supervisão on-line se mostre acompanhada de informações e de reflexão acerca das possibilidades e limitações do uso das TICs no contexto da supervisão (Tarlow et al., 2020; Twist et al., 2016).

Assim como a supervisão presencial, a supervisão remota contribui para o desenvolvimento da identidade profissional (Perry, 2012), favorece o desenvolvimento do senso de competência e de autoconfiança (Twist et al., 2016) e permite o estabelecimento de uma relação próxima entre supervisor e terapeuta (Lenz et al., 2011; Tarlow et al., 2020). Em um estudo com terapeutas de casal e família que passaram da supervisão presencial para a on-line, não se identificaram prejuízos à aliança de trabalho entre supervisores e terapeutas, nem aos níveis de satisfação com o processo de supervisão (Tarlow et al., 2020). A supervisão on-line também favorece o processo de autorreflexividade ao exigir maior preparo para as sessões (Conn et al., 2009; Rousmaniere et al., 2014), além de permitir o contato entre terapeutas que atuam em contextos diversos, aumentando o leque de experiências com que os supervisionandos têm contato e contribuindo para o desenvolvimento de uma perspectiva multicultural (Borcsa \& Pomini, 2017; Twist et al., 2016). A supervisão remota leva, ainda, à familiarização dos terapeutas com as TICs, o que favorece a qualidade da terapia on-line ofertada (Conn et al., 2009; Tarlow et al., 2020).

No entanto, a supervisão on-line também apresenta desafios que se mostram muito semelhantes àqueles enfrentados em relação à terapia on-line: a) o risco de quebra do sigilo e da confidencialidade relacionado ao uso das TICs; b) a quebra de fronteiras entre o espaço pessoal e o profissional, que pode levar supervisores e terapeutas a exporem sua privacidade e a terem a sensação de estarem constantemente trabalhando, o que aumenta sua vulnerabilidade ao burnout, c) maior dificuldade para o supervisor compreender o caso e orientar o terapeuta, caso não conheça a realidade de onde ele vive; d) ansiedade e temor vivenciados por terapeutas sem familiaridade com as TICs utilizadas na supervisão; e) dificuldades na comunicação relacionadas a questões técnicas, tais como atraso na resposta e falta de clareza na imagem; f) dificuldade em acessar e compreender sinais não verbais, o que pode passar a impressão de distanciamento e falta de reciprocidade (Borcsa \& Pomini, 2017; Kanz, 2001; Lenz et al., 2011; Martin, Lizarondo, \& Kumar, 2018; Rousmaniere et al., 2014; Tarlow et al., 2020).

Como forma de lidar com esses desafios, foram propostas orientações para a condução de supervisões on-line, que incluem: a) a obtenção de consentimento informado do terapeuta supervisionando e dos clientes atendidos; b) o estabelecimento de metas e a explicitação das expectativas de ambas as partes (supervisor e terapeuta); c) a definição de papéis, atribuições e responsabilidades; d) a atenção do supervisor à sua própria qualificação no que se refere aos conhecimentos sobre as plataformas utilizadas, os métodos de supervisão remota e a rede do município em que o terapeuta atende; e) o cuidado com o sigilo e a confidencialidade das informações de terapeutas e clientes (Bacigalupe, 2010; Borcsa \& Pomini, 2018; Kanz, 2001; Martin et al., 2018; Stretch, Nagel, \& Anthony, 2013).

De acordo com a revisão sistemática da literatura conduzida por Martin et al. (2018), os fatores que influenciam a qualidade da supervisão on-line podem ser divididos em grupos, que incluem as características do supervisor, as características do supervisionando, as características da supervisão, a relação entre o supervisor e o supervisionando, as estratégias comunicacionais utilizadas, os fatores ambientais e as questões técnicas. A supervisão on-line tem mais chances de se mostrar bem-sucedida quando o supervisionando opta por essa modalidade de supervisão, apresenta experiência clínica e conhece seu processo de aprendizagem e suas necessidades de supervisão. Por outro lado, o supervisor contribui para o sucesso da supervisão on-line quando conhece o contexto em que o supervisionando atua e se mostra disponível aos supervisionandos (Martin et al., 2018). Demonstrações de abertura, empatia e encorajamento também são valorizadas pelos supervisionandos (Lenz et al., 2011). 
Sessões de supervisão estruturadas, previamente organizadas e que se adequem às necessidades dos supervisionandos têm mais chances de serem bem-sucedidas, assim como aquelas em que cada um respeita o momento de o outro falar e em que as pessoas se expressam de maneira a favorecer sua compreensão (p. ex., fala mais lenta, uso de um estilo mais formal) (Martin et al., 2018). Também se mostram úteis encontros presenciais prévios e o uso de sessões mais frequentes no início da supervisão, para que se promova a confiança e a proximidade entre os membros do grupo. O tempo tende a contribuir para que sejam superadas as dificuldades na comunicação e no manejo das TICs.

\section{Considerações Finais}

O presente estudo sistematizou conhecimentos sobre terapia on-line com casais e famílias, trazendo considerações para a prática e a formação profissional no contexto da pandemia de Covid-19. Em particular, apresentamos e discutimos as potencialidades e os desafios para a utilização das TICs, sob o ponto de vista técnico, ético e formativo. Por se tratar de uma revisão narrativa da literatura, sugere-se a necessidade de estudos que investiguem evidências de eficácia e efetividade da terapia on-line com casais e famílias no Brasil, país em que essa modalidade de intervenção permanecia pouco explorada até a pandemia.

São diversos os desafios, de ordem profissional e pessoal, que a Covid-19 acrescenta à prática clínica com casais e famílias. Como profissionais, impõe-se a necessidade de buscar e aprender novos recursos que garantam o exercício eficaz do trabalho. Como pessoas, revela-se a demanda de flexibilidade para se adaptar à nova realidade, da qual terapeutas de casal e família também estão sofrendo as consequências, da mesma forma que seus clientes. Aqueles que atendem casais e famílias sabem que crises são momentos de perigo e/ou oportunidade. Sendo assim, a forma de enfrentamento profissional e pessoal desse momento crítico pode trazer excelentes avanços técnicos e de compreensão das relações conjugais e familiares, assim como de resiliência pessoal. O self do terapeuta de casal e família é instrumento essencial para o exercício de seu trabalho. Assim, eis na pandemia uma oportunidade de revisão e reflexão da prática clínica e da formação profissional de terapeutas de casal e família.

\section{Referências}

American Psychological Association. (2020). Office and Technology Checklist for Telepsychological Services. https://www.apa.org/practice/programs/dmhi/research-information/telepsychology-services-checklist.pdf

Anthony, K. (2000). Counselling in cyberspace. Counselling Journal, 11(10), 625-627.

Associação Brasileira de Terapia Familiar. (2018). Regimento interno da ABRATEF. ABRATEF. http://abratef.org.br/ 2019/wp-content/uploads/2019/09/RegimentoInternoAbratef-02-08-2018.pdf

Bacigalupe, G. (2010). Supervision 2.0: E-supervision a decade later. Bulletin, Family Therapy Magazine, 9(1), 38-41. https://www.researchgate.net/publication/220020816_Supervision_20_e-supervision_a_decade_later

Barak, A., Hen, L., Boniel-Nissim, M., \& Shapira, N. (2008). A comprehensive review and a meta-analysis of the effectiveness of internet-based psychotherapeutic interventions. Journal of Technology in Human Services, 26(2-4), 109-160. https://doi.org/10.1080/15228830802094429

Békés, V., \& Doorn, K. A. (2020). Psychotherapists' attitudes toward online therapy during the Covid-19 pandemic. Journal of Psychotherapy Integration, 30(2), 238-247. https://doi.org/10.1037/int0000214

Berger, T. (2017). The therapeutic alliance in internet interventions: A narrative review and suggestions for future research. Psychotherapy Research, 27(5), 511-524. https://doi.org/10.1080/10503307.2015.1119908

Blumer, M. L. C., Hertlein, K. M., \& VandenBosch, M. L. (2015). Towards the development of educational core competencies for couple and family therapy technology practices. Contemporary Family Therapy, 37, 113-121. https://doi.org/10.1007/s10591-015-9330-1

Borcsa, M., \& Pomini, V. (2017). Virtual relationships and systemic practices in the digital era. Contemporary Family Therapy, 39, 239-248. https://doi.org/10.1007/s10591-017-9446-6

Borcsa, M., \& Pomini, V. (2018). Couple and family therapy in the digital era. In J. Lebow, A. Chambers, \& D. C. Breunlin (Eds.), Encyclopedia of couple and family therapy (pp. 1-9). Springer. https://doi. org/10.1007/978-3-319-15877-8_1069-1 
Bouchard, S., Paquin, B., Payeur, R., Allard, M., Rivard, V., Fournier, T., Renaud, P., \& Lapierre, J. (2004). Delivering cognitive-behavior therapy for panic disorder with agoraphobia in videoconference. Telemedicine Journal and E-health, 10(1), 13-25. https://doi.org/10.1089/153056204773644535

Caldwell, B. E., Bischoff, R. J., Derigg-Palumbo, K. A., \& Liebert, J. D. (2017). Best practices in the on-line practice of couple and family therapy: Report of the online therapy workgroup. American Association for Marriage and Family Therapy.

Cartreine, J. A., Ahern, D. K., \& Locke, S. E. (2010). A roadmap to computer-based psychotherapy in the United States. Harvard Review of Psychiatry, 18(2), 80-95. https://doi.org/10.3109/10673221003707702

Conn, S. R., Roberts, R. L., \& Powell, B. M. (2009). Attitudes and satisfaction with a hybrid model of counseling supervision. Educational Technology \& Society, 12(2), 298-306. www.jstor.org/stable/jeductechsoci.12.2.298

Conselho Federal de Psicologia. (2012). Resolução CFP no 011/2012.. https://site.cfp.org.br/wp-content/uploads/ 2012/07/Resoluxo_CFP_nx_011-12.pdf

Conselho Federal de Psicologia. (2013). Carta de serviços sobre estágios e serviços-escola. https://site.cfp.org.br/ publicacao/carta-de-servicos-sobre-estagios-e-servicos-escola/

Conselho Federal dePsicologia. (2018). Resolução CFPno011/2018.https://e-psi.cfp.org.br/resolucao-cfp-no-11-2018/

Conselho Federal de Psicologia. (2020a). Resolução CFP $n^{\circ}$ 04/2020. https://www.in.gov.br/en/web/dou/-/ resolucao-n-4-de-26-de-marco-de-2020-250189333

Conselho Federal de Psicologia. (2020b). Práticas de estágios remotos em Psicologia no contexto da pandemia da Covid-19. https://site.cfp.org.br/cfp-e-abep-lancam-recomendacoes-sobre-praticas-e-estagios-remotos-emtempos-de-pandemia/

Coyne, L. W., Gould, E. R., Grimaldi, M., Wilson, K. G., Baffuto, G., \& Biglan, A. (2020). First things first: Parent psychological flexibility and self-compassion during Covid-19. Behavior Analysis in Practice. https://doi.org/10.1007/ s40617-020-00435-w

Faria, G. M. (2019). Constituição do vínculo terapêutico em psicoterapia online: Perspectivas gestálticas. Revista do NUFEN: Phenomenology and Interdisciplinarity, 11(3), 66-92. http://pepsic.bvsalud.org/ scielo.php?script=sci_arttext \&pid=S2175-25912019000300006

Ferrari, R. (2015). Writing narrative style literature reviews. Medical Writing, 24(4), 230-235. https://doi.org/10.1179/ 2047480615Z.000000000329

Fletcher-Tomenius, L., \& Vossler, A. (2009). Trust in online therapeutic relationships: The therapist's experience. Counselling Psychology Review, 24(2), 24-34.

Fortim, I. F., \& Cosentino, L. A. M. (2007). Serviço de orientação via e-mail: Novas considerações. Psicologia: Ciência e Profissão, 27(1), 164-175. http://pepsic.bvsalud.org/scielo.php?script=sci_arttext \&pid=S1414-98932007000100014

Glueckauf, R. L., Maheu, M. M., Drude, K. P., Wells, B. A., Wang, Y., Gustafson, D. J., \& Nelson, E. L. (2018). Survey of psychologists' telebehavioral health practices: Technology use, ethical issues, and training needs. Professional Psychology: Research and Practice, 49(3), 205-219. https://doi.org/10.1037/pro0000188

Gritti, A., Salvati, T., Russo, K., \& Catone, G. (2020). Covid-19 pandemic: A note for psychiatrists and psychologists. Journal of Psychosocial Systems, 4(1), 63-77. https://doi.org/10.23823/jps.v4il.70

Gritti, P. (2020). Family systems in the era of Covid-19: From openness to quarantine. Journal of Psychosocial Systems, 4(1), 1-5. https:// doi.org/10.23823/jps.v4i1.64

Grohol, J. M. (2004). Online counseling: A historical perspective. In R. Kraus, J. S. Zack, \& G. Stricker (Eds.), On-line counseling: A handbook for mental health professionals (pp. 51-68). Elsevier.

Hertlein, K. M., Blumer, M. L. C., \& Mihaloliakos, J. H. (2015). Marriage and family counselors' perceived ethical issues related to online therapy. The Family Journal, 23(1), 5-12. https://doi.org/10.1177/ 1066480714547184

Hertlein, K. M., Blumer, M. L. C., \& Smith, J. M. (2014). Marriage and family therapists' use and comfort with online communication with clients. Contemporary Family Therapy, 36, 58-69. https://doi.org/10.1007/s10591-013-9284-0

Himle, J. A., Fischer, D. J., Muroff, J. R., Van Etten, M. L., Lokers, L. M., Abelson, J. L., \& Hanna, G. L. (2006). Videoconferencing based cognitive-behavioral therapy for obsessive-compulsive disorder. Behaviour Research and Therapy, 44(12), 1821-1829. https://doi.org/10.1016/j.brat.2005.12.010 
Inchausti, F, MacBeth, A., Hasson-Ohayon, I., \& Dimaggio, G. (2020). Telepsychotherapy in the age of Covid-19: A commentary. Journal of Psychotherapy Integration, 30(2), 394-405. http://dx.doi.org/10.1037/int0000222

Kanz, J. E. (2001). Clinical-supervision.com: Issues in the provision of online supervision. Professional Psychology: Research and Practice, 32(4), 415-420. https://doi.org/10.1037/0735-7028.32.4.415

Lebow, J. L. (2020). Family in the age of Covid-19. Family Process, 59(2), 309-312.https://doi.org/10.1111/famp.12543

Lenz, A. S., Oliver, M., \& Nelson, K. W. (2011). In-person and computer-mediated distance group supervision: A case study. https://www.counseling.org/resources/library/vistas/2011-V-Online/Article_67.pdf

Linhares, M. B. M., \& Enumo, S. R. F. (2020). Reflexões baseadas na psicologia sobre efeitos da pandemia Covid-19 no desenvolvimento infantil. Estudos de Psicologia (Campinas), 37. https://doi.org/10.1590/1982-0275202037e200089

Lombard, M., \& Ditton, T. (1997). At the heart of it all: The concept of presence. Journal of Computer Mediated Communication, 3(2). https:// doi.org/10.1111/j.1083-6101.1997.tb00072.x

Lustgarten, S. D., Garrison, Y. L., Sinnard, M. T., \& Flynn, A. W. P. (2020). Digital privacy in mental healthcare: Current issues and recommendations for technology use. Current Opinion in Psychology, 36, 25-31. https://doi.org/10.1016/j.copsyc.2020.03.012

Machado, D., Laskoski, P. B., Severo, C. T., Bassols, A. M. S., Sfoggia, A., Kowacs, C., Krieger, D. V., Torres, M. B., Gastaud, M. B., Wellausen, R. S., Teche, S., \& Eizirik, C. L. (2016). A psychodynamic perspective on a systematic review of online psychotherapy for adults. British Journal of Psychotherapy, 32(1), 79-108. https://doi.org/10.1111/bjp.12204

MacMullin, K., Jerry, P., \& Cook, K. (2020). Psychotherapist experiences with telepsychotherapy: Pre Covid-19 lessons for a post Covid-19 world. Journal of Psychotherapy Integration, 30(2), 248-264. http://dx.doi.org/10.1037/ int0000213

Marques, E. S., Moraes, C. L., Hasselmann, M. H., Deslandes, S. F., Reichenheim, M. E. (2020). A violência contra mulheres, crianças e adolescentes em tempos de pandemia pela Covid-19: Panorama, motivações e formas de enfrentamento. Cadernos de Saúde Pública, 36(4). https://doi.org/10.1590/0102-311x00074420

Martin, P., Lizarondo, L., \& Kumar, S. (2018). A systematic review of the factors that influence the quality and effectiveness of telesupervision for health professionals. Journal of Telemedicine and Telecare, 24(4), 271-281. https://doi.org/10.1177/1357633X17698868

Matheson, B. E., Bohon, C., \& Lock, J. (2020). Family-based treatment via videoconference: Clinical recommendations for treatment providers during Covid-19 and beyond. International Journal of Eating Disorders, 53(7), 1142-1154. https://doi.org/10.1002/eat.23326

McCord, C. E., Console, K., Jackson, K., Palmiere, D., Stickley, M., Williamson, M. L. C., \& Armstrong, T. W. (2020). Telepsychology training in a public health crisis: A case example. Counselling Psychology Quarterly. https://doi.org/10.1080/09515070.2020.1782842

Oliveira, T. C., Abranches, M. V., \& Lana, R. M. (2020). (In)Segurança alimentar no contexto da pandemia por SARS-CoV-2. Cadernos de Saúde Pública, 36(4). https:// doi.org/10.1590/0102-311x00055220

Pennington, M., Patton, R., Ray, A., \& Katafiasz, H. (2017). A brief report on the ethical and legal guides for technology use in marriage and family therapy. Journal of Marital and Family Therapy, 43(4), 733-742. https://doi.org/10.1111/jmft.12232

Perry, C. W. (2012). Constructing professional identity in an online graduate clinical training program: Possibilities for online supervision. Journal of Systemic Therapies, 31(3), 53-67. https://doi.org/10.1521/jsyt.2012.31.3.53

Pickens, J. C., Morris, N., \& Johnson, D. J. (2020). The digital divide: Couple and family therapy programs' integration of teletherapy training and education. Journal of Marital and Family Therapy, 46(2), 186-200. https://doi.org/10.1111/jmft.12417

Pieta, M.A. (2014). Psicoterapia pelainternet:A relação terapêutica[Tese de Doutorado não-publicada]. Universidade Federal do Rio Grande do Sul.

Prime, H., Wade, M., \& Browne, D. T. (2020). Risk and resilience in family well-being during the Covid-19 pandemic. American Psychologist, 75(5), 631-643. https://doi.org/10.1037/amp0000660 
Proudfoot, J., Klein, B., Barak, A., Carlbring, P., Cuijpers, P., Lange, A., Ritterband, L., \& Andersson, G. (2011). Establishing guidelines for executing and reporting internet intervention research. Cognitive Behaviour Therapy, 40(2), 82-97. https://doi.org/10.1080/16506073.2011.573807

Ribeiro, M.S.A. (2005). Telesaúdenoensinoe pesquisaem psicologia clínica [Dissertação de Mestrado não-publicada]. Universidade Federal de Santa Catarina.

Richards, D., \& Vigano, N. (2013). Online Counseling: A narrative and critical review of the literature. Journal of Clinical Psychology, 69(9), 994-1011. https://doi.org/10.1002/jclp.21974

Roddy, M. K., Rothman, K., Cicila, L. N., \& Doss, B. D. (2019). Why do couples seek relationship help online? Description and comparison to in-person interventions. Journal of Marital and Family Therapy, 45(3), 369-379. https://doi.org/10.1111/jmft.12329

Rodrigues, C. G. (2014). Aliança terapêutica na psicoterapia breve on-line [Dissertação de Mestrado não-publicada]. Universidade de Brasília.

Roesler, C. (2017). Tele-analysis: The use of media technology in psychotherapy and its impact on the therapeutic relationship. Journal of Analytical Psychology, 62(3), 372-394. https://doi.org/10.1111/1468-5922.12317

Rousmaniere, T., Abbass, A., \& Frederickson, J. (2014). New developments in technology-assisted supervision and training: A practical overview. Journal of Clinical Psychology, 70(11), 1082-1093. https://doi.org/10.1002/jclp.22129

Schmidt, B., Crepaldi, M. A., Bolze, S. D. A., Neiva-Silva, L., \& Demenech, L. M. (2020). Saúde mental e intervenções psicológicas diante da pandemia do novo coronavírus (Covid-19). Estudos de Psicologia (Campinas), 37. https://doi.org/10.1590/1982-0275202037e200063

Silva, I. M., Lordello, S. R., Schmidt, B., \& Mieto, G. S. M. (2020). Brazilian families facing the Covid-19 outbreak. Journal of Comparative Family Studies, 51(3-4), 324-336. https://doi.org/10.3138/jcfs.51.3-4.008

Simpson, S. (2003). Video counselling and psychotherapy in practice. In S. Goss \& K. Anthony (Eds.), Technology in counselling and psychotherapy: A practitioner's guide (pp. 109-128). Palgrave Macmillan.

Simpson, S. G., \& Reid, C. L. (2014). Therapeutic alliance in videoconferencing psychotherapy: A review. Australian Journal of Rural Health, 22(6), 280-299. https:// doi.org/10.1111/ajr.12149

Skinner, A., \& Zack, J. S. (2004). Counseling and the internet. American Behavioral Scientist, 48(4), 434-446. https://doi.org/10.1177/0002764204270280

Stretch, L. S., Nagel, D. M., \& Anthony, K. (2013). Ethical for the use of technology in supervision. Therapeutic Innovations in Light of Technology, 3(2), 39-45. https://www.onlinetherapyinstitute.com/ ethical-framework-technology-supervision/

Tarlow, K. R., McCord, C. E., Nelon, J. L., \& Bernhard, P. A. (2020). Comparing in-person supervision and telesupervision: A multiple baseline single-case study. Journal of Psychotherapy Integration, 30(2), 383-393. https://doi.org/10.1037/int0000210

Twist, M. L. C., Hertlein, K. M., \& Haider, A. (2016). Electronic communication in supervisory relationships: A mixed data survey. Contemporary Family Therapy, 38, 424-433. https:// doi.org/10.1007/s10591-016-9391-9

United Nations Children's Fund. (2020). Covid-19: More than 95 per cent of children are out of school in Latin America and the Caribbean. https://www.unicef.org/press-releases/covid-19-more-95-cent-children-are-out-school-latinamerica-and-caribbean

Wade, S. L., Gies, L. M., Fisher, A. P., Moscato, E. L., Adlam, A. R., Bardoni, A., Corti, C., Limond, J., Modi, A. C., \& Williams, T. (2020). Telepsychotherapy with children and families: Lessons gleaned from two decades of translational research. Journal of Psychotherapy Integration, 30(2), 332-347. http://dx.doi.org/10.1037/int0000215

World Health Organization. (2020, 11 de agosto). WHO Coronavirus Disease (Covid-19) Dashboard. https://covid19.who.int/

Wrape, E. R., \& McGinn, M. M. (2019). Clinical and ethical considerations for delivering couple and family therapy via telehealth. Journal of Marital and Family Therapy, 45(2), 296-308. https://doi.org/10.1111/jmft.12319

\section{Beatriz Schmidt}

Psicóloga, Mestre e Doutora em Psicologia. Professora do Curso de Graduação em Psicologia e do Programa de PósGraduação em Psicologia da Universidade Federal do Rio Grande, Rio Grande - RS. Brasil.

E-mail: psi.beatriz@gmail.com

(1) https://orcid.org/0000-0003-2907-2297 
Isabela Machado da Silva

Psicóloga, Mestre e Doutora em Psicologia. Professora do Curso de Graduação em Psicologia e do Programa de PósGraduação em Psicologia Clínica e Cultura da Universidade de Brasília, Brasília - DF. Brasil.

E-mail: isabela.ms@gmail.com

(1) https://orcid.org/0000-0002-9654-9342

\section{Maria Adélia Minghelli Pieta}

Psicóloga, Mestre e Doutora em Psicologia. Pesquisadora no Programa de Pós-Graduação em Psicologia da Universidade Federal do Rio Grande do Sul, Porto Alegre - RS. Brasil.

E-mail: mampieta@gmail.com

(1) https://orcid.org/0000-0002-2084-7554

\section{Maria Aparecida Crepaldi}

Psicóloga, Mestre em Psicologia Clínica e Doutora em Saúde Mental. Professora do Programa de Pós-Graduação em Psicologia da Universidade Federal de Santa Catarina, Florianópolis - SC. Brasil.

E-mail: maria.crepaldi@gmail.com

(1) https://orcid.org/0000-0002-5892-7330

\section{Adriana Wagner}

Psicóloga, Doutora em Psicologia Social. Professora do Programa de Pós-Graduação em Psicologia da Universidade Federal do Rio Grande do Sul, Porto Alegre - RS. Brasil.

E-mail: adrianaxwagner@gmail.com

(1) https://orcid.org/0000-0002-0629-2310

Endereço para envio de correspondência:

Universidade Federal do Rio Grande (FURG). Av. Itália, Km 8, Instituto de Ciências Humanas e da Informação, Curso de Psicologia, Bairro Carreiros. CEP: 96201-900. Rio Grande - RS. Brasil.

Recebido 19/08/2020

Aceito 01/09/2020

Received 08/19/2020

Approved 09/01/2020

Recibido 19/08/2020

Aceptado 01/09/2020

Como citar: Schmidt, B., Silva, I. M., Pieta, M. A. M., Crepaldi, M. A., Wagner, A. (2020). Terapia on-line com casais e famílias: Prática e formação na pandemia de Covid-19. Psicologia: Ciência e Profissão, 40, 1-15. https://doi.org/10.1590/1982-3703003243001

How to cite: Schmidt, B., Silva, I. M., Pieta, M. A. M., Crepaldi, M. A., Wagner, A. (2020). Online therapy with couples and families: Practice and training in the Covid-19 pandemic. Psicologia: Ciência e Profissão, 40, 1-15. https://doi.org/10.1590/1982-3703003243001

Cómo citar: Schmidt, B., Silva, I. M., Pieta, M. A. M., Crepaldi, M. A., Wagner, A. (2020). Terapia de parejas y familias on line: Práctica y formación en la pandemia de Covid-19. Psicologia: Ciência e Profissão, 40, 1-15. https://doi.org/10.1590/1982-3703003243001 